\title{
Are patient-reported outcome measures the way to go in inflammatory bowel disease?
}

\author{
Subrata Ghosh MBBS MD FRCPC FRCP FRCPE, Editor-in-Chief
}

$\mathrm{T}$ here is general agreement that clinical disease activity indexes in inflammatory bowel disease require improvement. The clinical disease activity indexes were composite scores designed to be used as efficacy measures in randomized controlled clinical trials; however, some of them are also used sporadically in clinical practice. More recently, the clinical disease activity indexes have been regularly supported by more objective measures of inflammatory activity such as endoscopic scores, and/or C-reactive protein, fecal calprotectin levels, etc. In addition, patient-reported disease specific or generic quality of life scores have been regularly used to support clinical efficacy of a candidate drug in trials, although these are not widely used in clinical practice. All of these have been the cornerstone of drug development in inflammatory bowel disease, despite their imperfections (1).

The composite clinical disease activity indexes include patientreported items, physician-reported items, laboratory measures and, in some cases, endoscopy scores in various permutations and combinations, and the different items may be unweighted or weighted. These activity indexes generally contain several subjective items prone to imprecision, and may drive up placebo response scores unpredictably, depending on the time point of assessment. In addition, the physicianreported items may be subject to interpretation and prone to distortion in clinical trial settings. Endoscopy scores are also physician reported and, hence, there is an increasing trend toward centralized readouts to have more consistent scoring of endoscopic appearance. In pediatric inflammatory bowel disease, the clinical disease activity indexes are often modified for this specific age group.

In the current issue of the Journal, El-Matary (2) (pages 536-542) has provided an excellent review of the evolution of patient-reported outcome measures in inflammatory bowel disease and the various regulatory agency recommendations driving changes in the way we measure the efficacy of drugs in clinical trials. In addition, there is an increasing desire among physicians caring for inflammatory bowel disease patients to incorporate scoring systems in their clinical practice to introduce more objectivity in their assessment of patients. Electronic medical records are incorporating such scoring systems in their physician input interface. Various electronic platforms are being designed for real-time capture of patient-reported outcome measures. These are encouraging developments in improving the overall standard of care in inflammatory bowel disease.

However, there are significant challenges and hurdles to be overcome to introduce patient-reported outcome measures widely in inflammatory bowel disease and eliminate physician-reported outcomes. The heterogeneity of inflammatory bowel disease presentations is remarkable, and symptoms may arise from a multitude of contributing and confounding factors. These factors are highlighted in Box 1, although the list is by no means exhaustive. These may vary at different phases of disease and may be coloured by previous experience, quality of communication with the physician and members of the multidisciplinary team, as well as other factors such as occupation and education level of the patient, family support and belief systems. While some of these may be controlled by inclusion and exclusion criteria in clinical trials, as well as by the randomization process, there is little doubt that not all factors can be controlled. Combining patient-reported outcome measures with objective measures, such as endoscopic appearance (itself a physician-reported item, even in central reporting systems), may be more robust, although we still need more information regarding the exact operating characteristics in large multicentre clinical trials. In clinical practice, the heterogeneity can be enormous and well-trained physician input is essential. Therefore, we must find a balance among patient-reported outcomes, physician assessment, laboratory parameters and other objective measures, such as cross-sectional imaging and endoscopy (with inherent interobserver agreement issues), to make the correct decisions. Although significantly more work must be done, replacing the physician is not an option.

\section{BOX 1}

Contributors to patient-reported outcome measures in inflammatory bowel disease that are not directly related to inflammatory activity

- Irritable bowel syndrome/visceral hypersensitivity

- Complications such as strictures or fistulae or neoplasm

- Loss of function (eg, colon) due to chronic inflammation

- Concomitant infections

- Psychosocial issues, including anxiety and depression

- latrogenic factors, medical or surgical

- Bile acid malabsorption

- Small bowel bacterial overgrowth

- Extraintestinal manifestations not related to inflammatory activity

- Alternative medical care

- Health care and family/friends support system of the patient

\section{REFERENCES}

1. Dassopoulos T, Sultan S, Falck-Ytter YT, Inadomi JM, Hanauer SB. American Gastroenterological Association Institute technical review on the use of thiopurines, methotrexate, and anti-TNF- $\alpha$ biologic drugs for the induction and maintenance of remission in inflammatory Crohn's disease. Gastroenterology 2013;145:1464-78.e1-5.

2. El-Matary W. Patient reported outcome measures in inflammatory bowel disease. Can J Gastroenterol Hepatol 2014;28:536-42. 


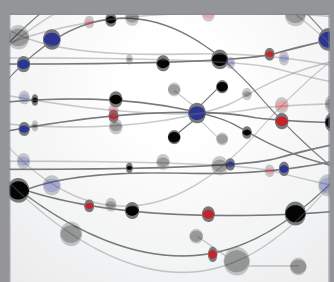

The Scientific World Journal
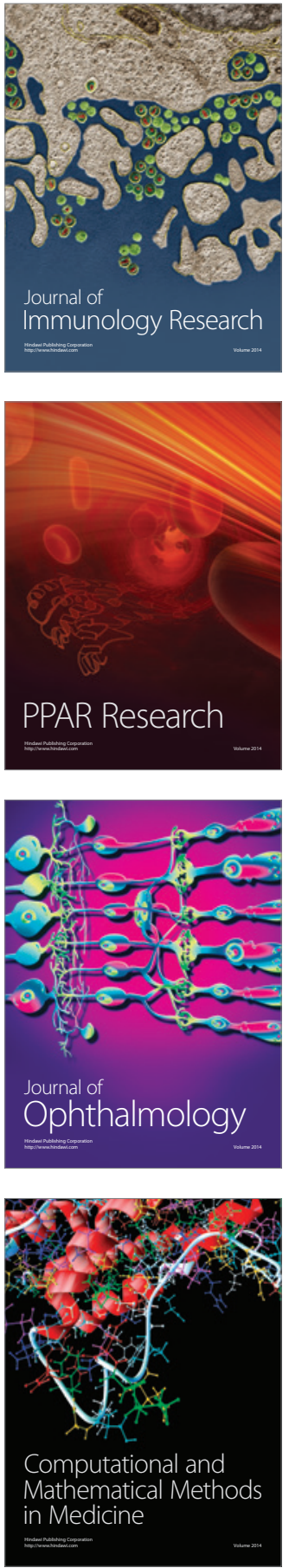

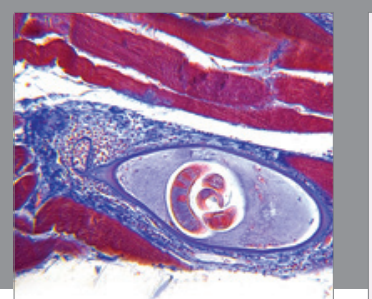

Gastroenterology Research and Practice

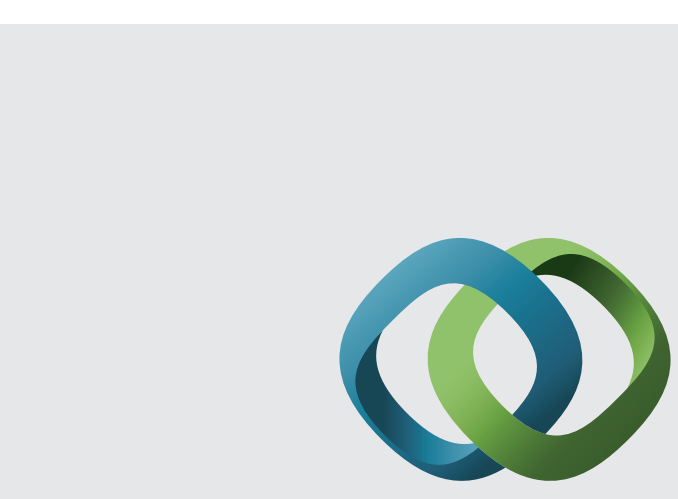

\section{Hindawi}

Submit your manuscripts at

http://www.hindawi.com
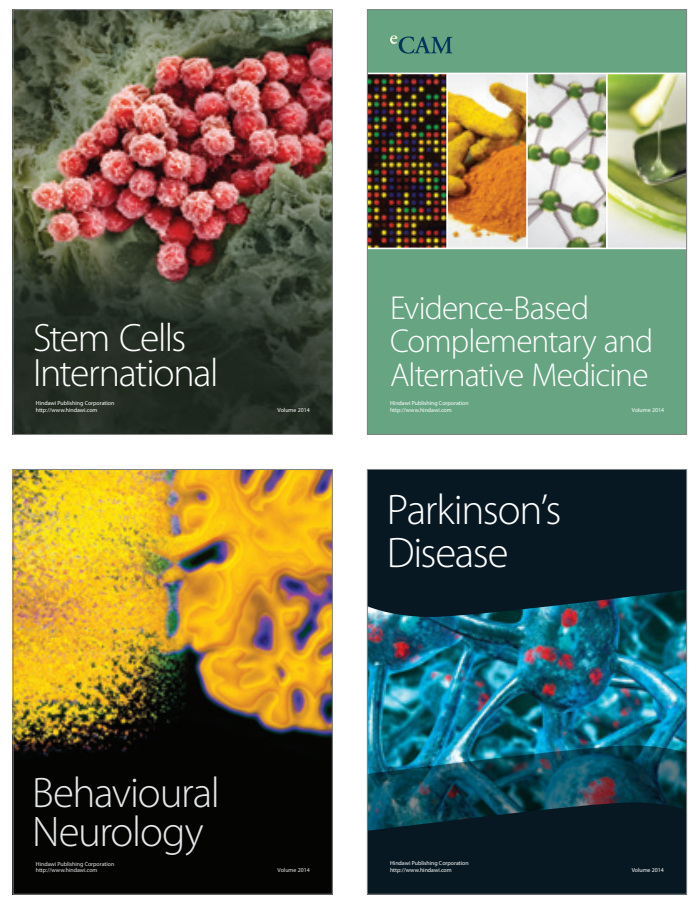
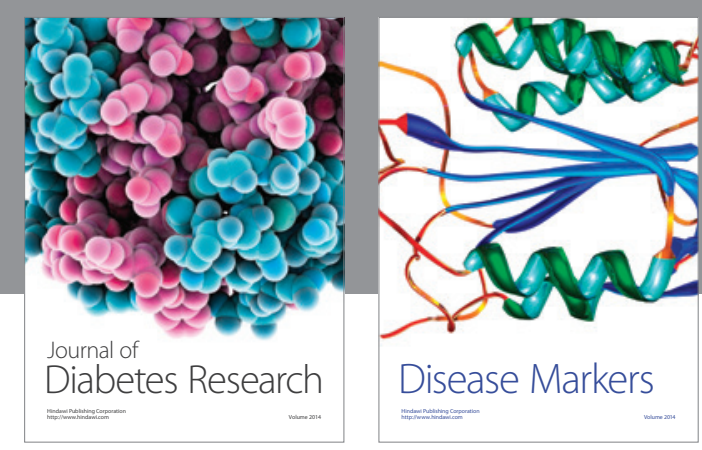

Disease Markers
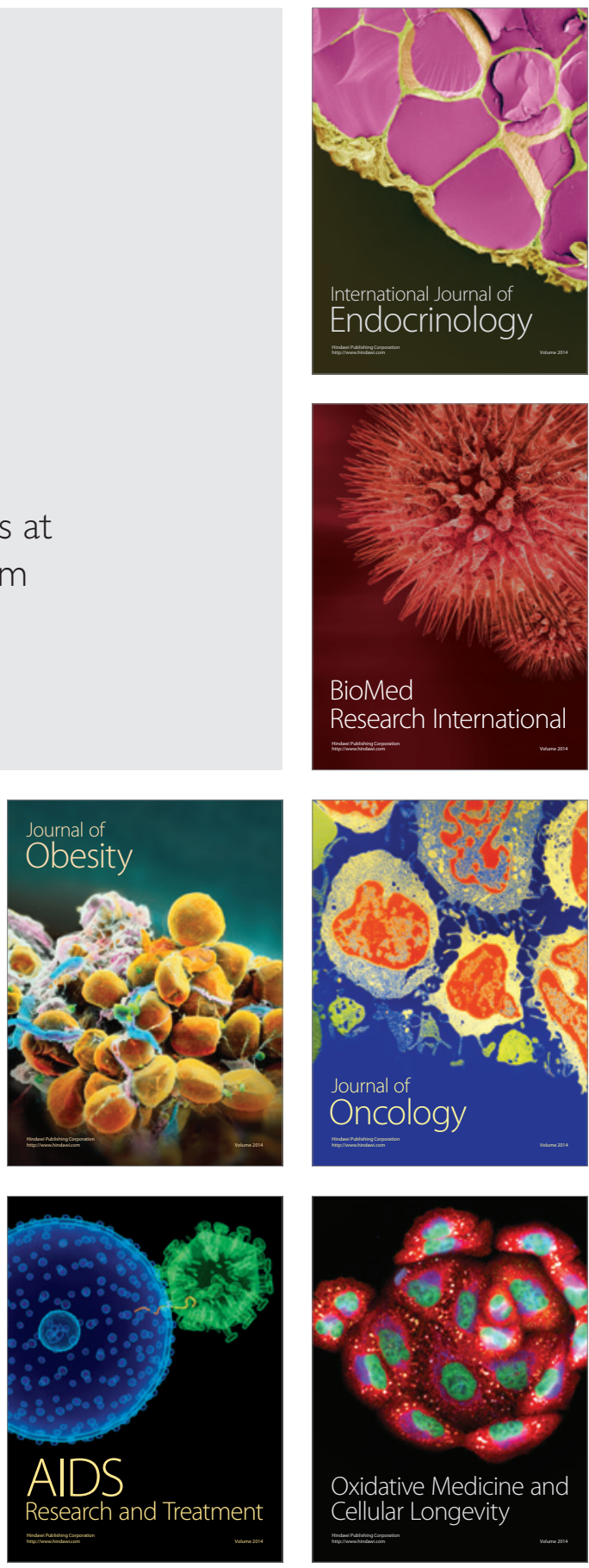